\title{
Patient age, tumor appearance and tumor size are risk factors for early recurrence of cervical cancer
}

\author{
JUAN WANG, TAO WANG, YUN-YI YANG, YAN-LAN CHAI, FAN SHI and ZI LIU \\ Department of Radiation Oncology, The First Affiliated Hospital of Xi'an Jiaotong University \\ School of Medicine, Xi'an, Shanxi 710061, P.R. China
}

Received September 18, 2014; Accepted November 14, 2014

DOI: $10.3892 / \mathrm{mco} .2014 .465$

\begin{abstract}
The recurrence and metastasis of cervical cancer contribute to a poor prognosis. The aim of this study was to investigate the risk factors for cervical cancer progression. A total of 284 patients with recurrent cervical cancer were retrospectively recruited to evaluate the association of disease recurrence with clinicopathological data. The univariate analysis demonstrated that patient age, tumor appearance and tumor size were significantly associated with early recurrence and metastasis of the disease $(\mathrm{P}<0.05)$. However, clinical stage, tumor histology, pathological stage and initial treatment options were not associated with early recurrence and metastasis of cervical cancer $(\mathrm{P}>0.05)$. The multivariate analysis also demonstrated that patient age, tumor appearance and tumor size were independent risk factors for the early recurrence of cervical cancer $(\mathrm{P}<0.05)$. Therefore, these three factors should be taken into consideration in the management of cervical cancer.
\end{abstract}

\section{Introduction}

Cervical cancer is the second most common and the fifth deadliest malignancy among women worldwide (1-3). Patients with recurrent cervical cancer exhibit a very high mortality, despite several currently available treatment options, although central-local recurrences, most likely due to a better clinical response to chemoradiation therapy, have a better overall survival rate compared to lateral-pelvic recurrences (45.4 vs. $18.2 \%$, respectively) (4-6). Previous studies have demonstrated that lymph node metastasis, parametrial and surgical margin involvement, tumor size and deep stromal invasion, but not histological type or grade, signifi-

Correspondence to: Dr Zi Liu, Department of Radiation Oncology, The First Affiliated Hospital of Xi'an Jiaotong University School of Medicine, 277 West Yanta Road, Xi'an, Shanxi 710061, P.R. China E-mail: liuzimai12014@163.com

Key words: cervical cancer, recurrence, concurrent chemoradiotherapy, radical surgery cantly affect cervical cancer recurrence (7-10). However, the risk factors that are considered to contribute to cervical cancer recurrence are not universally accepted or duplicated in other study populations. Furthermore, the risk factors contributing to early recurrence $(<2$ years) have not been investigated, although recurrence and metastasis of cervical cancer contribute to a poor prognosis. Other molecular analyses have demonstrated that altered expression of different genes predicts early recurrence of cervical cancer (7-10), but these may not be superior to routine clinicopathological parameters (7-10). Thus, in this study, we aimed to perform a retrospective analysis of 284 cervical cancer patients with disease recurrence in order to determine the association of their clinicopathological parameters with early cervical cancer recurrence.

\section{Patients and methods}

Study population. A total of 284 patients with cervical cancer were enrolled at the Department of Radiation Oncology, The First Affiliated Hospital of Xi'an Jiaotong University School of Medicine (Xi'an, China) between May, 2005 and April, 2008. The patients were treated in our hospital and the inclusion criteria included complete medical records, confirmed pathological diagnosis, known histological grade at initial treatment, first radical treatment regimen and known recurrence and metastasis. This study was approved by the Institutional Review Board of our hospital and written informed consent was obtained from each participant.

Definition of patients with uncontrolled, recurrent and metastatic disease. The cervical cancer patients underwent radical surgery and tumor lesions were completely removed (microscopic resection margins free of tumor cells). Disease recurrence and metastasis were defined as the presence of pelvic and distant tumor lesions, respectively, 1 year after surgery. If the recurrence or metastasis occurred within 1 year, it was defined as uncontrolled disease. Furthermore, after the cervical cancer patients were treated by radiation therapy, if a tumor lesion was identified in the cervix, pelvic region, or distant site after wound healing at 3 months after radiotherapy, it was defined as recurrent or metastatic disease; if a tumor lesion was found within 3 months, it was defined as uncontrolled disease. 
Diagnosis of patients with uncontrolled, recurrent and metastatic disease. The diagnosis of local recurrence after radiotherapy was mainly based on clinical and pathological examinations. Specifically, the diagnosis of pelvic recurrence following radiotherapy mainly depends on the presence of pelvic masses or nodular thickening by uterine examination, which is aggravated by pain and other symptoms (such as lower limb edema and positive findings after radiographic examination). The diagnosis of pelvic recurrence after surgery was mainly based on the occurrence of a tumor lesion at the operation site and field following pelvic, cytological and imaging examination. However, the diagnosis of distant metastasis mainly relies on clinical examination, computed tomography, magnetic resonance imaging, positron emission tomography/computed tomography, or other imaging examinations, as well as tumor biopsy.

Statistical analysis. The statistical analysis was performed using SPSS software, version 13.0 (SPSS Inc., Chicago, IL, USA). The univariate analysis was performed using the Chi-squared test or Fisher's exact test, while the multivariate analysis used the Cox regression model. $\mathrm{P}<0.05$ was considered to indicate a statistically significant difference.

\section{Results}

Univariate analysis of factors contributing to uncontrolled, recurrent and metastatic cervical cancer. The clinicopathological characteristics of the patients are shown in Table I, including patient age, clinical stage, tumor appearance, tumor histology, pathological stage, tumor size and initial treatment options. The univariate analysis demonstrated that patient age, tumor appearance and tumor size were significantly associated with early recurrence and metastasis of the disease $(\mathrm{P}<0.05)$. However, clinical stage, tumor histology, pathological stage and initial treatment options were not associated with early recurrence and metastasis of cervical cancer $(\mathrm{P}>0.05)$.

Multivariate analysis to identify independent risk factors contributing to uncontrolled, recurrent and metastatic disease. Furthermore, we performed a multivariate analysis to identify potential independent risk factors that contribute to early recurrence and metastasis of cervical cancer and found that patient age, tumor appearance and tumor size were independent factors associated with early recurrence of cervical cancer (Table II).

\section{Discussion}

Cervical cancer remains a significant public health concern worldwide $(2,5,6)$, particularly among women aged $\leq 65$ years or in developing countries (11-13). Approximately one-third of patients with invasive, recurrent, or metastatic cervical cancer eventually succumb to the disease. The recurrence rate of cervical cancer ranges from $\sim 10 \%$ for International Federation of Gynecology and Obstetrics stages Ib-IIa to $\sim 60 \%$ in locally advanced cases (stages IIb-IVa) (14). The mortality rate in patients with early recurrence is even higher (14). Previous studies identified several risk factors, such as tumor size and lymph node metastasis, which are associated with the recurrence of cervical cancer (7-10). Consistently, in our present study, we identified tumor size as an independent risk factor associated with early recurrence of cervical cancer. Furthermore, our data identified patient age and tumor appearance as independent risk factors contributing to early recurrence of cervical cancer.

Over the last few years, the incidence of cervical cancer has increased among younger women; furthermore, younger patients with cervical cancer have a worse clinical outcome $(15,16)$. Our present findings demonstrated that patient age was an independent determining factor for early recurrence of cervical cancer, which was consistent with the findings of previous studies $(15,16)$. Compared to older patients, younger cervical cancer patients often exhibit the following characteristics: immune deficiency; tobacco smoking; high serum hormone levels; cervical erosions; HPV16 infection; and high levels of survivin, cyclooxygenase 2, matrix metalloproteinase and CD44 expression. In addition, younger patients with cervical cancer also exhibit poor tumor differentiation and lymph node metastasis. These characteristics may collectively contribute to early recurrence of cervical cancer in this patient population. Thus, the age of the patient requires careful consideration in the treatment of cervical cancer.

Previous studies demonstrated that a cervical cancer lesion with a diameter $>4 \mathrm{~cm}$ is more difficult to control compared to smaller lesions $(17,18)$. The reason may be that large tumor lesions are more frequently associated with an earlier onset of distant tumor metastasis $(17,18)$. Large cervical cancer lesions with a cauliflower-like and ulcerative tumor appearance often lack sufficient blood supply in their lesion center; thus, they subsequently recruit hypoxic cells and are more resistant to radiation therapy. Furthermore, exophytic cervical cancers often destroy the normal appearance of the cervix, which is likely to affect the pelvic radiation dose distribution and reduce the effective radiation dose administered to the tumor cells. All these factors may result in difficult control of these large, exophytic, ulcerated cervix cancers, subsequently causing early recurrence or metastasis. Therefore, in accordance with the 2012 National Comprehensive Cancer Network Cervical Cancer Clinical Practice Guidelines, such patients should be treated by radical surgery with chemoradiotherapy. Chemotherapy enhances the radiosensitivity of tumor cells for better local control and also controls potential distant tumor metastasis. If the patients fail to show local control following radiotherapy, surgical removal of local tumor lesions should be considered, which may help control tumor progression, reduce recurrence and improve patient outcome (19-21). These reasons may explain why patient age, tumor appearance and tumor size are independent factors for cervical cancer recurrence and they should be considered for future management of cervical cancer. However, several factors, such as patient age and tumor size, stage and localization, may also affect the outcome or therapeutic decisions made in cervical cancer patients. In this patient cohort, only patient age and tumor appearance and size were identified as independent predictors of recurrence and metastasis of cervical cancer, whereas treatment selection (radiation vs. surgery) and clinicopathological stage did not predict recurrence or metastasis of cervical cancer. Thus, future studies are required to verify our findings prior to their application in clinical practice. 
Table I. Univariate analysis of association of clinicopathological parameters with uncontrolled, recurrent and metastatic cervical cancer.

\begin{tabular}{|c|c|c|c|}
\hline Characteristics & $\begin{array}{l}\text { Patient no. } \\
(\mathrm{n}=284)\end{array}$ & $\begin{array}{c}\text { Ratio of early } \\
\text { recurrence, n (\%) }\end{array}$ & P-value \\
\hline Age (years) & & & 0.049 \\
\hline$\leq 35$ & 19 & $17(89.47)$ & \\
\hline$>35$ & 265 & $180(67.92)$ & \\
\hline Clinical stage & & & 0.053 \\
\hline IA & 10 & $3(30.00)$ & \\
\hline IB & 47 & $31(65.95)$ & \\
\hline IIA & 78 & $60(76.92)$ & \\
\hline IIB & 84 & $61(72.61)$ & \\
\hline IIIA & 9 & $5(55.56)$ & \\
\hline IIIB & 56 & $37(66.07)$ & \\
\hline Tumor appearance & & & 0.038 \\
\hline Ulcerative & 8 & $7(87.51)$ & \\
\hline Endocervical & 37 & $31(83.78)$ & \\
\hline Exophytic & 221 & $150(67.87)$ & \\
\hline Erosive & 18 & $9(50.00)$ & \\
\hline Histology & & & 0.372 \\
\hline Squamous cell carcinoma & 249 & $175(70.28)$ & \\
\hline Non-squamous cell carcinoma & 35 & $22(62.85)$ & \\
\hline Pathological stage & & & 0.766 \\
\hline I & 22 & $16(72.73)$ & \\
\hline II & 214 & $146(68.22)$ & \\
\hline III & 48 & $35(72.91)$ & \\
\hline Tumor size $(\mathrm{cm})$ & & & 0.000 \\
\hline$\leq 4$ & 150 & $78(52.00)$ & \\
\hline$>4$ & 134 & $119(88.81)$ & \\
\hline Treatment & & & 0.969 \\
\hline Radiotherapy & 118 & $82(69.28)$ & \\
\hline Surgery & 166 & $115(69.49)$ & \\
\hline
\end{tabular}

Table II. Multivariate analysis of the association of clinicopathological parameters with uncontrolled, recurrent and metastatic cervical cancer.

\begin{tabular}{lcccccc}
\hline Parameters & B & SE & Wald & Df & P-value & Exp (B) \\
\hline Treatment options & 0.096 & 0.219 & 0.194 & 1 & 0.660 & 1.101 \\
Age & -0.790 & 0.264 & 8.975 & 1 & 0.003 & 0.454 \\
Clinical stage & -0.042 & 0.075 & 0.312 & 1 & 0.577 & 0.959 \\
Tumor appearance & -0.379 & 0.131 & 8.293 & 1 & 0.004 & 0.685 \\
Histology & -0.306 & 0.202 & 2.286 & 1 & 0.131 & 0.737 \\
Pathological stage & 0.235 & 0.162 & 2.120 & 1 & 0.145 & 1.265 \\
Tumor size & 0.510 & 0.158 & 10.432 & 1 & 0.001 & 1.665 \\
\hline
\end{tabular}

\section{Acknowledgements}

We would like to thank Professor Zhuang Gui Hua of the Department of Public Health, College of Medicine, Xi'an
Jiaotong University, for his assistance with the statistical analyses. This study was supported in part by a grant from the National Natural Science Foundation of China (no. 81071838). 


\section{References}

1. Armstrong EP: Prophylaxis of cervical cancer and related cervical disease: a review of the cost-effectiveness of vaccination against oncogenic HPV types. J Manag Care Pharm 16: 217-230, 2010 .

2. Peirson L, Fitzpatrick-Lewis D, Ciliska D and Warren R: Screening for cervical cancer: a systematic review and meta-analysis. Syst Rev 2: 35, 2013.

3. Priebe AM: 2012 Cervical cancer screening guidelines and the future role of HPV testing. Clin Obstet Gynecol 56: 44-50, 2013

4. Smaniotto D, D'Agostino G, Luzi S, et al: Concurrent 5-fluorouracil, mitomycin $\mathrm{C}$ and radiation with or without brachytherapy in recurrent cervical cancer: a scoring system to predict clinical response and outcome. Tumori 91: 295-301, 2005.

5. Peiretti M, Zapardiel I, Zanagnolo V, et al: Management of recurrent cervical cancer: a review of the literature. Surg Oncol 21: e59-e66, 2012.

6. Elst P, Ahankour F and Tjalma W: Management of recurrent cervical cancer. Review of the literature and case report. Eur J Gynaecol Oncol 28: 435-441, 2007.

7. Morley GW and Seski JC: Radical pelvic surgery versus radiation therapy for stage I carcinoma of the cervix (exclusive of microinvasion). Am J Obstet Gynecol 126: 785-798, 1976.

8. Hopkins MP and Morley GW: Radical hysterectomy versus radiation therapy for stage IB squamous cell cancer of the cervix. Cancer 68: 272-277, 1991.

9. Estape RE, Angioli R, Madrigal M, et al: Close vaginal margins as a prognostic factor after radical hysterectomy. Gynecol Oncol 68: 229-232, 1998.

10. Mabuchi S, Isohashi F, Yoshioka Y, et al: Prognostic factors for survival in patients with recurrent cervical cancer previously treated with radiotherapy. Int J Gynecol Cancer 20: 834-840 2010.

11. Scatchard K, Forrest JL, Flubacher M, et al: Chemotherapy for metastatic and recurrent cervical cancer. Cochrane Database Syst Rev 10: CD006469, 2012
12. Tao X, Hu W, Ramirez PT and Kavanagh JJ: Chemotherapy for recurrent and metastatic cervical cancer. Gynecol Oncol 110 (3 Suppl 2): S67-S71, 2008.

13. Moore DH: Chemotherapy for advanced, recurrent and metastatic cervical cancer. J Natl Compr Canc Netw 6: 53-57, 2008.

14. Benedet JL, Odicino F, Maisonneuve P, et al: Carcinoma of the cervix uteri. Int J Gynaecol Obstet 83 Suppl 1: 41-78, 2003.

15. Yang YC, Shen J, Tate JE, et al: Cervical cancer in young women in Taiwan: prognosis is independent of papillomavirus or tumor cell type. Gynecol Oncol 64: 59-63, 1997.

16. Cao L, Li X, Zhang Y and Wang Q: Clinical features and prognosis of cervical cancer in young women. J Central South Univ (Med Sci) 35: 875-878, 2010

17. Goncalves A, Fabbro M, Lhomme C, et al: A phase II trial to evaluate gefitinib as second- or third-line treatment in patients with recurring locoregionally advanced or metastatic cervical cancer. Gynecol Oncol 108: 42-46, 2008.

18. Keys HM, Bundy BN, Stehman FB, et al; Gynecologic Oncology Group: Radiation therapy with and without extrafascial hysterectomy for bulky stage IB cervical carcinoma: a randomized trial of the Gynecologic Oncology Group. Gynecol Oncol 89: 343-353, 2003.

19. Cetina L, Garcia-Arias A, Candelaria M, et al: Brachytherapy versus radical hysterectomy after external beam chemoradiation: a non-randomized matched comparison in IB2-IIB cervical cancer patients. World J Surg Oncol 7: 19, 2009.

20. Classe JM, Rauch P, Rodier JF, et al; Groupe des Chirurgiens de Centre de Lutte Contre le Cancer: Surgery after concurrent chemoradiotherapy and brachytherapy for the treatment of advanced cervical cancer: morbidity and outcome: results of a multicenter study of the GCCLCC (Groupe des Chirurgiens de Centre de Lutte Contre le Cancer). Gynecol Oncol 102: 523-529, 2006.

21. Morice P, Uzan C, Zafrani Y, et al: The role of surgery after chemoradiation therapy and brachytherapy for stage IB2/II cervical cancer. Gynecol Oncol 107 (1 Suppl 1): S122-S124, 2007. 